\title{
Genomic and transcriptomic insights into the ecology and metabolism of benthic archaeal cosmopolitan, Thermoprofundales (MBG-D archaea)
}

\author{
Zhichao Zhou ${ }^{1,3} \cdot$ Yang Liu ${ }^{1} \cdot$ Karen G. Lloyd $\mathbb{D}^{2} \cdot$ Jie Pan ${ }^{1} \cdot$ Yuchun Yang ${ }^{3} \cdot$ Ji-Dong Gu $\mathbb{D}^{3} \cdot$ Meng Li ${ }^{1}$
}

Received: 26 July 2018 / Revised: 7 October 2018 / Accepted: 4 November 2018 / Published online: 4 December 2018

(c) The Author(s) 2018. This article is published with open access

\begin{abstract}
Marine Benthic Group D (MBG-D) archaea, discovered by 16S rRNA gene survey decades ago, are ecologically important, yet understudied and uncultured sedimentary archaea. In this study, a comprehensive meta-analysis based on the 16S rRNA genes of MBG-D archaea showed that MBG-D archaea are one of the most frequently found archaeal lineages in global sediment with widespread distribution and high abundance, including 16 subgroups in total. Interestingly, some subgroups show significant segregations toward salinity and methane seeps. Co-occurrence analyses indicate significant non-random association of MBG-D archaea with Lokiarchaeota (in both saline and freshwater sediments) and Hadesarchaea, suggesting potential interactions among these archaeal groups. Meanwhile, based on four nearly complete metagenome-assembled genomes (MAGs) and corresponding metatranscriptomes reconstructed from mangrove and intertidal mudflat sediments, we provide insights on metabolic potentials and ecological functions of MBG-D archaea. MBG-D archaea appear to be capable of transporting and assimilating peptides and generating acetate and ethanol through fermentation. Metatranscriptomic analysis suggests high expression of genes for acetate and amino acid utilization and for peptidases, especially the M09Btype extracellular peptidase (collagenase) showing high expression levels in all four mangrove MAGs. Beyond heterotrophic central carbon metabolism, the MBG-D genomes include genes that might encode two autotrophic pathways: WoodLjundahl (WL) pathways using both $\mathrm{H}_{4} \mathrm{MPT}$ and $\mathrm{H}_{4}$ folate as $\mathrm{C}_{1}$ carriers, and an incomplete dicarboxylate/4-hydroxybutyrate cycle with alternative bypasses from pyruvate to malate/oxaloacetate during dicarboxylation. These findings reveal MBG-D archaea as an important ubiquitous benthic sedimentary archaeal group with specific mixotrophic metabolisms, so we proposed the name Thermoprofundales as a new Order within the Class Thermoplasmata. Globally, Thermoprofundales and other benthic archaea might synergistically transform benthic organic matter, possibly playing a vital role in sedimentary carbon cycle.
\end{abstract}

Supplementary material The online version of this article (https:// doi.org/10.1038/s41396-018-0321-8) contains supplementary material, which is available to authorized users.

Ji-Dong Gu

jdgu@hku.hk

$\triangle$ Meng Li

limeng848@szu.edu.cn

1 Institute for Advanced Study, Shenzhen University, 518060 Shenzhen, People's Republic of China

2 Department of Microbiology, University of Tennessee, Knoxville, TN 37996, USA

3 Laboratory of Environmental Microbiology and Toxicology, School of Biological Sciences, The University of Hong Kong, Pokfulam Road, Hong Kong SAR, Hong Kong, People's Republic of China

\section{Introduction}

Archaea in the subsurface ecosystem play crucial roles in global biogeochemical cycles. The estimated global subsurface sedimentary microbial abundance reaches $2.9 \times$ $10^{29}$, comprising around $9.1-31.5 \%$ of the total number of prokaryotes on the Earth [1]. Recent studies highlighted the vast deposit of archaeal cellular biomass in marine subsurface sediments buried to a depth of $>1 \mathrm{~m}$ in a wide range of oceanographic settings [2]. Cell membrane lipid studies also show evidence of more living archaea than bacteria [2] and archaea possessing the active metabolic capacity to assimilate sedimentary organic compounds [3]. Within these subsurface environmental settings, the general archaeal cosmopolitans, such as Marine Benthic Group B (MBG-B), Marine Benthic Group D (MBG-D), and Bathyarchaeota, 
are dominant archaeal groups, which might contribute significantly to biogeochemical cycles [3-6]. MBG-D archaea have long been recognized from 16S rRNA gene surveys in benthic environments, and their global distribution and abundance place them as universal players in both terrestrial and marine subsurface realms (Supplementary Table S1) [5, 7-9]. DNA-based 16S rRNA gene community analyses suggest that MBG-D archaea have specific environmental niches and co-occurrence patterns. They co-occur with anaerobic methanotrophic archaea in methane-driven seeps [10], are abundant in liquid $\mathrm{CO}_{2}$ or $\mathrm{CO}_{2}$ hydrate-bearing marine sediments [10], and their 16S rRNA gene abundance appears to be independent of biogeochemical zones of sulfate reduction and methanogenesis [2, 5, 11]. Furthermore, MBG-D archaea are also found to progressively replace methanogens going downcore in samples from a freshwater lake [12], and they are also abundant and persistent in hypersaline environments and exhibit small variations of community composition correlated with the change of carbon content [13].

In recent years, genome contents and metabolic pathways of MBG-D archaea have been explored using singlecell genomic and metagenomic approaches [5, 14]. MBG-D archaea are thought to be benthic anaerobic archaea capable of exogenous protein mineralization and acetogenesis [5, 14]. They could secrete active extracellular peptidases in marine sediments [5]. Furthermore, a metagenomic survey reveals that MBG-D archaea and Bathyarchaeota co-exist in White Oak River estuary sediments with high abundance, sharing similar inferred metabolic capacities for acetogenesis and protein degradation in estuarine organic-rich regimes $[14,15]$. Owing to their potential importance in carbon transformation and ubiquitous distribution, it is important to have a broader view of the ecological, genomic, and metabolic understanding of MBG-D archaea. However, the few available partial genomes limit our thorough understanding of their global ecological roles and metabolisms. The ecological diversity, genomic blueprints, metabolic properties, and biogeochemical functions of MBG-D archaea remain elusive, though MBG-D archaea have been identified for many years.

Here we conducted a comprehensive meta-analysis based on the available 16S rRNA gene sequences of MBG-D archaea to investigate their global environmental distribution, the environmental associations of different subgroups, and the potential synergistic relationship with other archaeal lineages. We also resolved four nearly complete MBG-D metagenome-assembled genomes (MAGs) from subsurface sediments of mangrove forests and intertidal mudflats in Mai Po Nature Reserve, Hong Kong and one additional MBG-D MAG from the publicly available dataset. These MAGs, together with their metatranscriptomes (Mai Po), provided a better insight on the active metabolic and ecological functions of MBG-D archaea. Based on the unique phylogenetic position and metabolic potentials, we proposed MBG-D archaea as a new order Thermoprofundales within the class Thermoplasmata. Finally, we also addressed the relationships of distribution patterns to metabolic capacities and proposed the potential biogeochemical roles of these ubiquitous sedimentary archaea in carbon cycling.

\section{Materials and methods}

\section{Sampling, nucleic acids extraction, and metagenome/metatranscriptome sequencing}

Sediment samples for DNA extraction were collected from Mai Po Nature Reserve on September 12, 2014. Mai Po Nature Reserve is characterized as a subtropical, coastal wetland with a variety of wetland types, such as intertidal mudflats, mangrove forest, shrimp ponds, and manmade fishery ponds [16]. One subsurface sediment sample (MaiPo-8) was collected from a site covered by mangrove forest $\left(22^{\circ} 29.875^{\prime} \mathrm{N}, 114^{\circ} 01.767^{\prime} \mathrm{E}\right)$ at a sediment depth of $10-15 \mathrm{~cm}$, and a deeper sediment sample (MaiPo-9, at $20-25 \mathrm{~cm}$ depth) was also collected at the same site. Another sediment sample (MaiPo-11) was collected from a nearby intertidal mudflat site $\left(22^{\circ} 29.949^{\prime} \mathrm{N}, 114^{\circ} 01.656^{\prime} \mathrm{E}\right)$ at a depth of $13-16 \mathrm{~cm}$, which was a more homogeneous fine slurry with more reduced redox state than the former two sediment samples. The detailed sampling descriptions and physicochemical parameters are listed in Supplementary Information Note 1 as well as in our previous studies $[17,18]$. Bulk sediment DNA (10 g) was isolated according to the manufacturer's instructions (DNeasy PowerMax Soil Kit, QIAGEN) and concentrated for metagenome sequencing (Novogene Inc., Beijing, China). The samples for metatranscriptomic analysis were also sampled from the same sites and layers as those used for metagenomes at a later time (details in Supplementary Information Note 1). The sediment samples were preserved immediately after sampling with the LifeGuard Soil Preservation Solution (QIAGEN) to prevent RNA degradation. Total RNA was isolated from bulk sediments (5-25 g) according to the manufacturer's instructions (RNeasy PowerSoil Total RNA Kit, QIAGEN). Genomic DNA was removed from total RNA (TURBO DNA-free Kit, Ambion, USA), and the remaining RNA was further concentrated (RNeasy MinElute Cleanup Kit, QIAGEN). The extracted RNA (with rRNA removed by Ribo-Zero rRNA Removal Kit, Illumina, USA) was subjected to metatranscriptomic sequencing in GENEWIZ Inc., Suzhou, China (details of library construction and sequencing in Supplementary Information Note 1). 


\section{Phylogenetic analysis of MBG-D archaeal 16S rRNA gene sequences}

A total of 3133 MBG-D archaeal 16S rRNA gene sequences (>1200 bps) were downloaded from SILVA SSURef 128 and parsed by a homemade Perl script to acquire their "isolation source" and "note" from corresponding gbk files [19]. The sequences were aligned by SINA [20], filtered by $50 \%$ sequence consensus and ssuref:archaea filters in ARB [21] (stored as "SSU_MBG-D.arb"), and dereplicated at the 97\% level by QIIME [22]. The remaining sequences were used to construct phylogenetic trees with Thermoplasma volcanium GSS1 as an outgroup by RAxML-HPC v8 on XSEDE (CIPRES gateway) using "-T 4 -f a -c 25 -N 1000 -m GTRCAT -p 12345 -x 12345” and IQ-TREE 1.5.5 (Web server) using "-st DNA -m GTR+G4+F -bb 1000 -alrt $1000 "$ " $[20,23,24]$. The final tree was visualized by iTOL [25]. Clades with $<0.36$ branch length were assigned as subgroups and supporting bootstrap values were also taken into consideration. The environmental category, salinity, and acidity conditions were acquired from the sequence metadata. A total of 8503 MBG-D 16S rRNA gene sequences ( $>900 \mathrm{bp}$ ) were downloaded from the SILVA SSURef 128 and assigned to subgroups in "SSU_MBG-D. arb" by the ARB parsimony quick-add method after sequence alignment and column filtering (the same aligning and filtering method as that in building the backbone tree described above) [21]. Sequences originating from one study were regarded as one library, and environmental information parsed from NCBI was assigned to each library. The indicator lineages (ILs; MBG-D subgroups) for environments were calculated by IndVal in R package labdsv [26], which combines relative abundance and relative frequency to identify indicators significantly associated with environments (only studies with $>5$ sequences were included). The relative abundances of ILs were visualized by "polarHistogram.R" (https://github.com/chrislad).

\section{Meta-analysis and community networks of MBG-D archaea from sediments}

Sediment archaeal 16S rRNA gene sequences were retrieved by "16S AND 600:2000 [Sequence Length] AND archaea [Organism] AND rrna [Feature key] AND isolation_source [All fields] NOT (genome OR chromosome OR plasmid)" in NCBI nucleotide database and aligned by SINA with SILVA taxonomy assigned (those without taxonomic assignments were excluded) [29,022 retrieved with 26,394 left after filtering (conducted on Nov 26, 2017)] [20]. Environmental conditions were assigned to individual studies according to sequence metadata in the same way as described above. QIIME scripts were used to make operational taxonomic unit (OTU) tables at $97 \%$ cutoff level with the default settings: one with each study having $>30$ sequences ("over30_OTU table" with 177 studies), one with each study having >10 MBG-D sequences ("MBGD_over10_OTU table" with 58 studies) (script details in Supplementary Information Note 2) [22]. Species abundance distribution (SAD) and index of dispersion (IoD) plots were calculated to reflect the occurrence and abundance pattern and the dispersion pattern of archaeal lineages, respectively, based on the "over30_OTU table." The beta-diversity patterns based on "MBG-D_over10_OTU table" were reflected in categories of salinity, environments, and seep condition, with 1000 permutations of the adonis test. Co-occurrence network analysis was performed according to the previous methods [27, 28] (details in Supplementary Information Note 3) based on "over30_OTU table." The observed network reflected positive correlations (edges) among OTUs (nodes) with Spearman's $\rho>0.4$ and Benjamini-Hochberg adjusted $p$ value $<0.01$. The observed network ended up with 205 nodes and 571 edges, and identically sized Erdös-Réyni (ER) random networks were simulated for 1000 times for comparison of network topological properties. The network construction, property characterization and visualization, and random network stimulation were conducted by $\mathrm{R}$ packages (vegan and igraph) and software Gephi [29-31].

\section{Metagenomic assembly, binning, and annotation}

The 150 bp pair-end raw reads from Illumina HiSeq were dereplicated by a Perl script implemented in SeqTools (Genome Research Ltd.), then subjected to Sickle to trim low-quality reads with the default settings (https://github. com/najoshi/sickle). Clean reads for individual samples were applied in idba v1.1.1 for de novo scaffold assembling separately [32], with the settings of "--mink 65 --maxk 145 --step 10." Obtained assemblies were deposited in the DOEJGI IMG database and annotated by the DOE-JGI Microbial Genome Annotation Pipeline (MGAP v.4) [33]. In the first step, initial binning was conducted by MaxBin v2.2.1 with the default setting and the minimum contig length as 1000 bp [34]. Then CheckM v1.0.7 was used to assess the completeness and contamination of all MAGs and provide the placement of MAGs in the concatenated marker protein tree [35]. Based on the taxonomic information given by the default reference genomes and MAGs or single-cell amplified genomes (SAGs) within Thermoplasmata in the concatenated marker protein tree, MAGs affiliated with the Thermoplasmata clade were picked. Next, all potential Thermoplasmata MAGs from three samples, together with other reference MBG-D MAGs, SAGs, and fosmids affiliated with Thermoplasmata, were used as the reference for mapping raw reads by BBmap using "minid" as 0.6 (details in Supplementary Information Note 4) [36]. The properly 
a

1st Ring

$\square$ acidic wetland sediment

$\square$ biomat

$\square$ brackish sediment/water

$\square$ carbonate spring

$\square$ cave water

$\square$ coastal sediment

$\square$ cold spring

$\square$ estuarine sediment

freshwater

$\square$ freshwater sediment

$\square$ groundwater

$\square$ hot spring

hydrothermal sediment

$\square$ lake sediment/column

$\square$ mangrove sediment

marine hydrothermal vent

marine sediment

$\square$ marine water column

$\square$ methane seep sediment

$\square$ mud volcano sediment

$\square$ oil reservoir

$\square$ soil

$\square$ uncharaterized

wetland sediment

2nd Ring

$\square$ Saline

$\square$ non-Saline

$\square$ Hypersaline

$\square$ not known

3rd Ring

$\square$ Seep

$\square$ non-Seep/not known

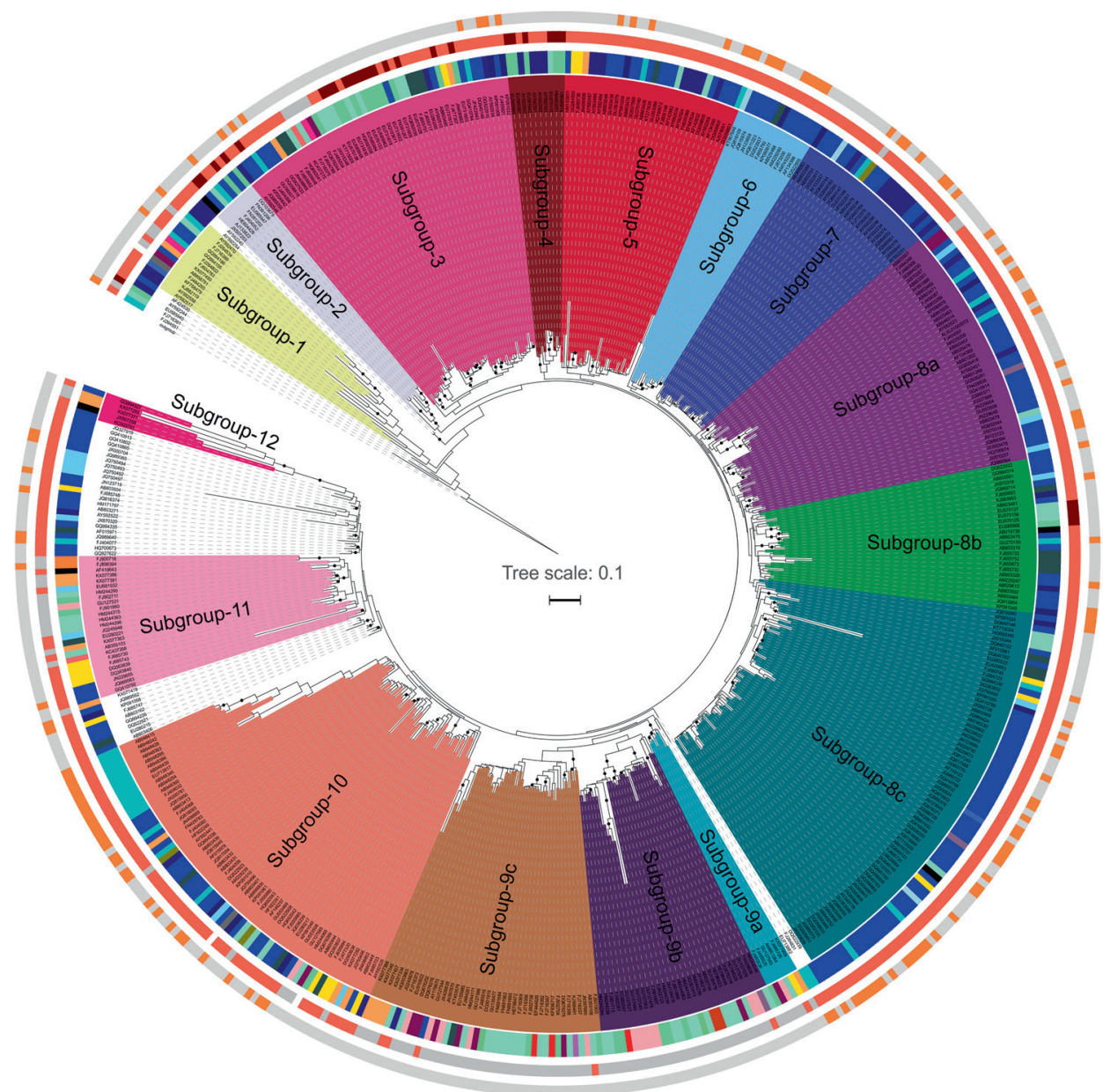

b

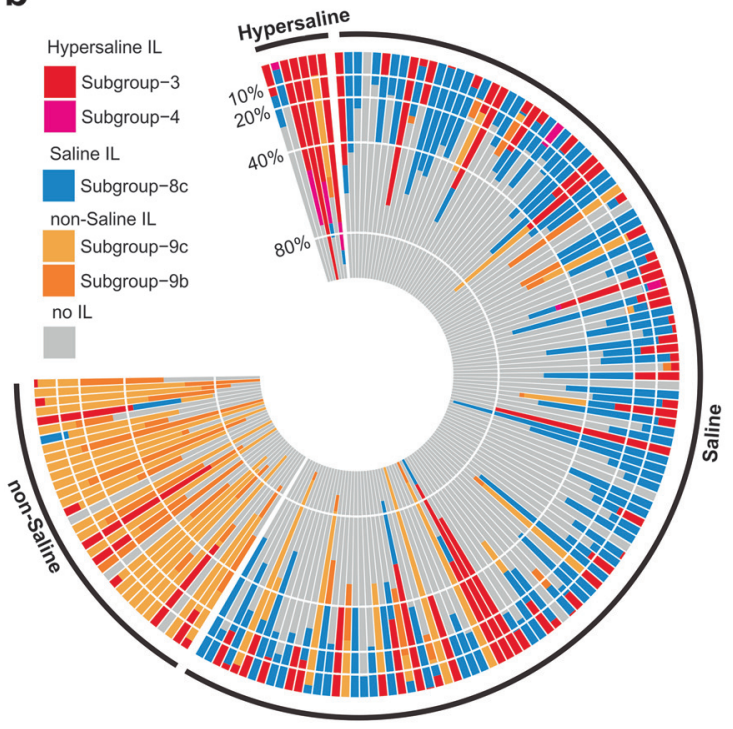

paired reads mapping on the reference were dereplicated and trimmed and subsequently re-assembled by the same method described above. MaxBin v2.2.1 was applied to bin
C
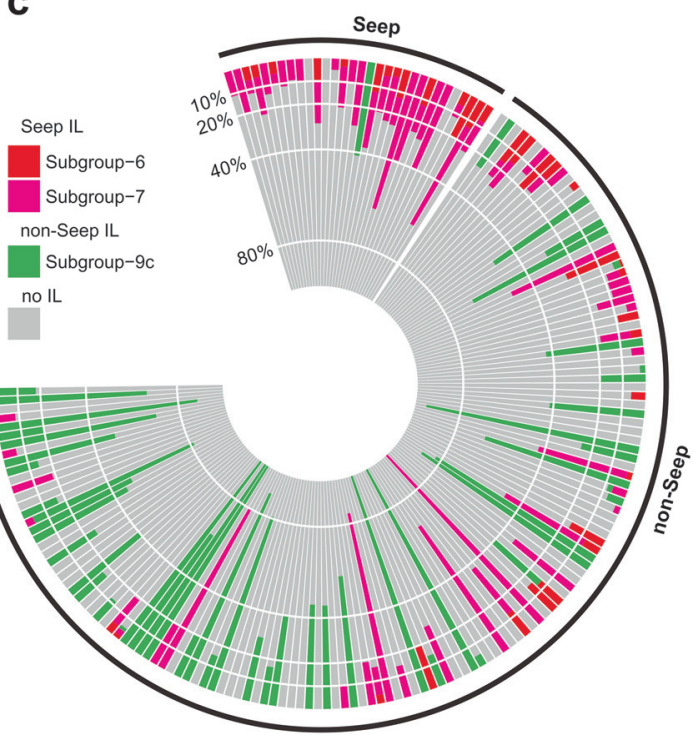

above sub-assemblies with the minimum contig length as 2000 bp. MAGs with $>50 \%$ completeness were used and manually curated to reduce the contamination and strain 
Fig. 1 Phylogenetic tree of Thermoprofundales (a) and relative abundances of indicator lineages associated with environments $(\mathbf{b}, \mathbf{c})$. Dereplicated Thermoprofundales sequences (97\% cutoff) from SILVA SSURef 128 was used to construct this RAxML-based phylogenetic tree. From the inside to the outside, the first ring denotes 25 environmental categories, the second ring denotes the salinity, and the third ring denotes the seep environment condition. Nodes with bootstrap values $>75 \%$ were marked with black dots. The outgroup is the $16 \mathrm{~S}$ rRNA gene sequence from Thermoplasma volcanium GSS1. The indicator lineages are inferred by their relative abundances and relative frequencies in all the libraries that they occur, based on statistical analysis. The significantly supported indicator lineages associated with salinity and seep conditions were used to plot the polar histogram figures depicting their abundance patterns in all the studied libraries

heterogeneity. Finally, MAGs were translated by Prodigal v2.6.3 and annotated by non-redundant NCBI protein database (NCBI nr database, updated by Oct 4, 2016), BlastKOALA, and EggNOG v4.5.1 (HMMER mapping mode) with default settings [37-40]. The peptidases were recognized by the MEROPS database and also confirmed by the annotation of Pfam using InterProScan v5.21-60.0 and the top hit result using the nr database [40-42]. Peptidases with extracellular signal peptides were predicted using POSRTb and PRED-SIGNAL, and only congruent results from both of them led to assigning an extracellular peptidase [43, 44].

\section{Phylogenetic analysis of MBG-D genomes}

The alignment of 43 concatenated phylogenomic markers, including all MBG-D MAGs and reference genomes (85 genomes in total), were processed by the CheckM software [35]. Only the MBG-D MAGs with completeness $>70 \%$ were considered, and concatenated alignment sequences with $<25 \%$ informative sites were excluded except for the reference genomes. Columns with $>90 \%$ gaps along the alignment were deleted. The refined concatenated alignment was subjected to RAxML-HPC BlackBox on XSEDE (CIPRES gateway) for phylogenomic tree construction with a bacterial genome (Acidimicrobium ferrooxidans DSM 10331) as the outgroup and using "-m PROTCATLG -f a -N autoMRE"' settings [23, 45].

The alignment of $16 \mathrm{~S}$ rRNA genes was double filtered by 50\% MBG-D sequence consensus and ssuref:archaea filters in ARB [21]. The phylogenetic tree of $16 \mathrm{~S}$ rRNA genes was constructed by RAxML-HPC BlackBox on XSEDE (CIPRES gateway) using "-m GTRCAT -f a -N autoMRE" settings (details could be found in Supplementary Information Note 2).

\section{Metatranscriptomic analysis}

Potential rRNA reads from raw data were filtered by SortMeRna v2.1b with the default settings [46]. Non-rRNA transcripts of individual samples were mapped to corresponding open-reading frames of MBG-D MAGs by Bowtie2 v2.2.8 with settings of "--no-mixed --no-discordant --no-dovetail --no-contain --no-overlap --very-sensitive." The subsequent gene coverage of reads and Transcripts Per Kilobase Million (TPM) were calculated by "pileup.sh" (BBmap) [36] and "TPM-RPKM-calculator.py" (https:// github.com/RichieJu520). TPM allowed comparisons of gene expression from sample to sample by normalizing different sequencing depths. The metatranscriptome from each sample was mapped to its corresponding MAGs, respectively.

\section{Results and Discussion}

\section{Ecological significance of MBG-D archaea}

The phylogenetic tree of MBG-D archaea was reconstructed using 508 OTU representatives at $97 \%$ cutoff value, with 91.5\% sequences assigned to 16 subgroups (Fig. 1a). MBG$\mathrm{D}$ archaea have wide distribution with $16 \mathrm{~S}$ rRNA gene sequences originated from 25 environmental categories. Among them, the top 3 most abundant environments were marine sediments, marine hydrothermal vents, and mangrove sediments, accounting for approximately $70 \%$ of the total sequences currently available in the database (Supplementary Table S1). The phylogenetic trees of subgroups are roughly congruent in the RAxML and IQ trees (Supplementary Information Note 2), indicating that subgroup topology remains largely stable, even with partial branch nodes with low bootstrap support. The IndVal function identifies the ILs (MBG-D subgroups) not only significantly associate with particular environments $(p$ value $<0.05)$ but also constitute a large fraction of the lineages in their respective environments (Fig. 1b, c). The significant segregation of subgroups toward saline and seep condition echoes the previous research on Bathyarchaeota, in which the distinct evolutionary Bathyarchaeota subgroups have been found in freshwater and marine sediments, suggesting a niche-specific adaptation [47].

\section{Meta-analysis of sedimentary MBG-D archaea}

An updated collection of 23,194 sedimentary archaeal sequences from 177 studies (each study contains at least 30 sequences) was assigned to 36 archaeal lineages for SAD analysis, plotting relative abundance against occurrence (frequency of archaeal lineage in all studies) (Fig. 2a, Supplementary Table S2). A linear regression with significant support indicates that archaeal lineages of widespread distribution across studies have higher abundance (frequency of occurrence in sequence collection) than those 
a

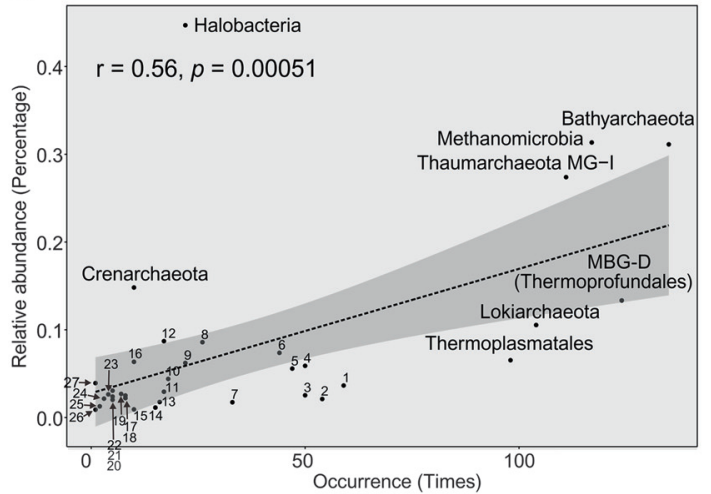

b

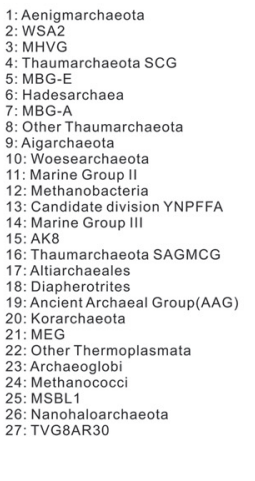

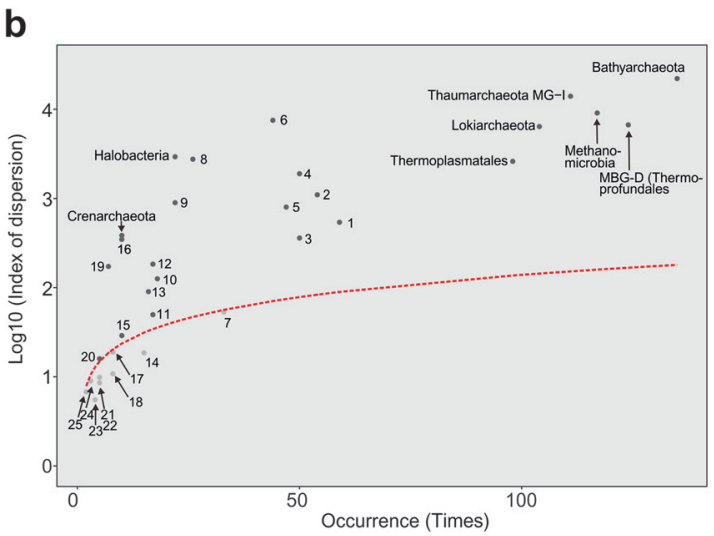

C
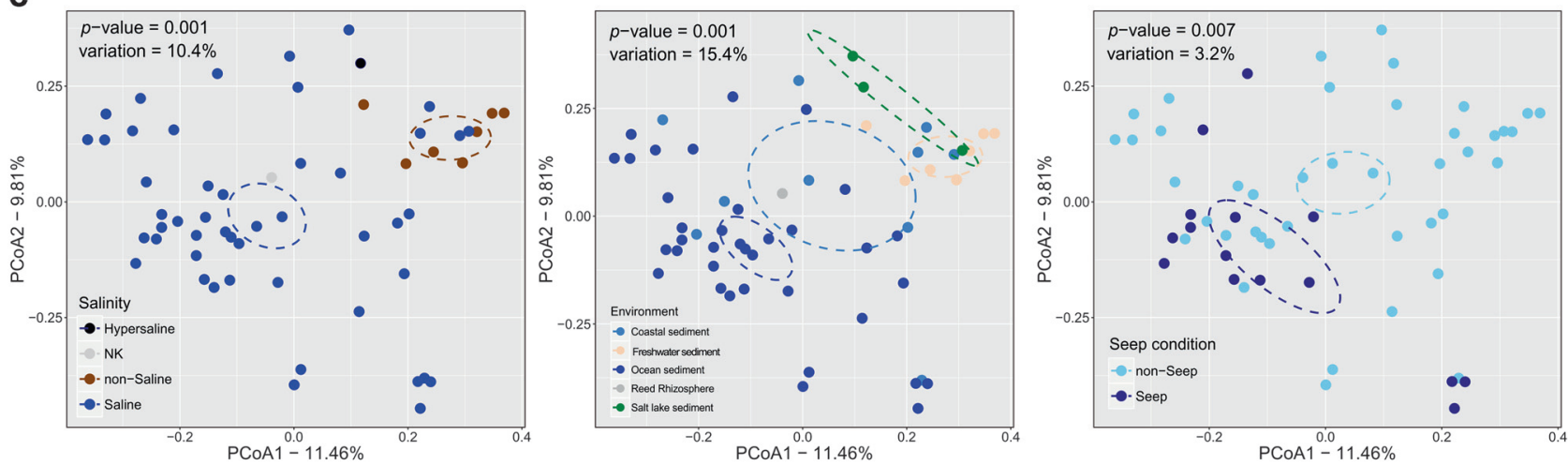

d

e
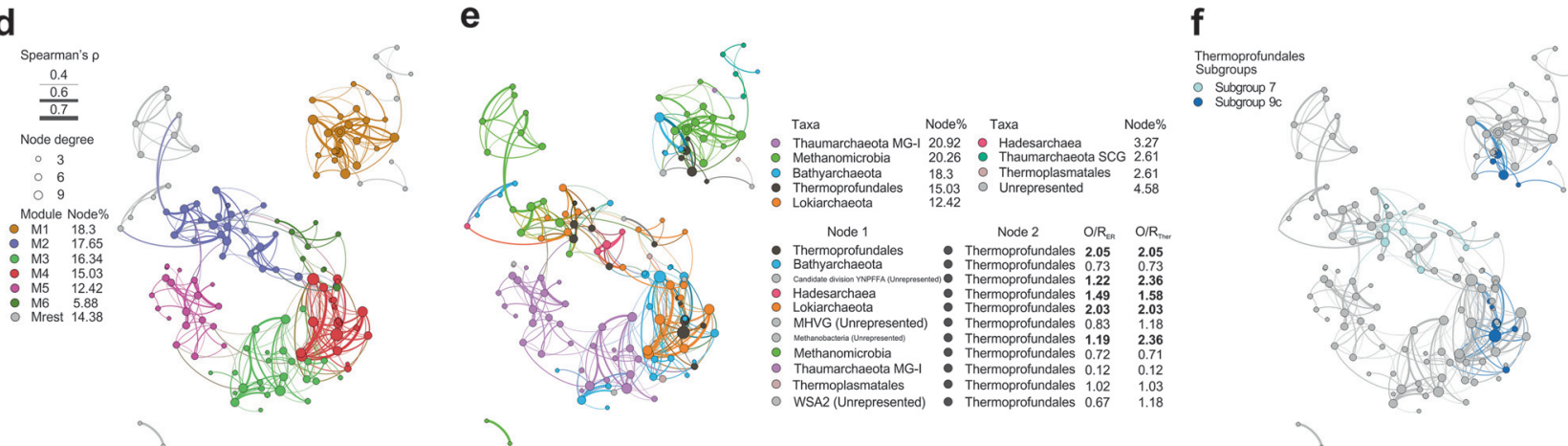

Fig. 2 a Species abundance distribution (SAD) figure with relative abundances of archaeal lineages plotted against their occurrences in 177 studies ("over30_OTU table"). The vertical axis stands for the average relative abundance of one archaeal lineage across all libraries that they appear; the horizontal axis stands for the number of times this archaeal lineage being detected across all libraries. b Index of dispersion (IoD) figure with $\log _{10}$-transformed indices of dispersion of archaeal lineages plotted against their occurrences in 177 studies ("over30_OTU table"). Taxa with singletons are excluded from the IoD figure. The red line depicts the $0.5 \%$ confidence limit of chisquare distribution. Lineages below this line follow a Poisson distribution and are randomly distributed in the environment. c Beta diversity plots of 58 studies based on the "MBG-D_over10_OTU table." Beta diversity was calculated by the unweighted Unifrac matrix method with a 1000-permutation adonis test. Subplots color-coded by salinity, environment, and seep condition are shown, with dashed-line

of limited distribution in environments, similar to the previous report [47]. The archaeal lineages could be divided circles representing 95\% confidence intervals for groupings. Cooccurrence networks depict correlations among nodes that are affiliated within different modules (d) and different archaeal lineages (e). Nodes affiliated to Thermoprofundales subgroups are highlighted in the network (f). The ratio of observed co-occurring incidence between two archaeal lineages $(O)$ over random co-occurring incidence $(R)$ of that pair $\left(R_{\mathrm{ER}}\right.$ is the mean value of the observed co-occurring incidences for 1000 identically sized Erdös-Réyni random networks; $R_{\text {Theo }}$ is the theoretical co-occurring incidence calculated by giving the identical frequencies of archaeal lineages and random association between nodes) is an estimation of non-random association of two archaeal lineages; that is $\mathrm{O} / \mathrm{R}$ ratio more than 1 stands for two archaeal lineages non-randomly associated (potentially reflecting a synergistic relationship) in the environment. Significant non-random associations of Thermoprofundales and other lineages are highlighted (with two $\mathrm{O} /$ $R$ ratios significantly $>1$ )

into two groups of over or under 75 occurrences, with one group as cosmopolitan lineages of persistent/abundant 


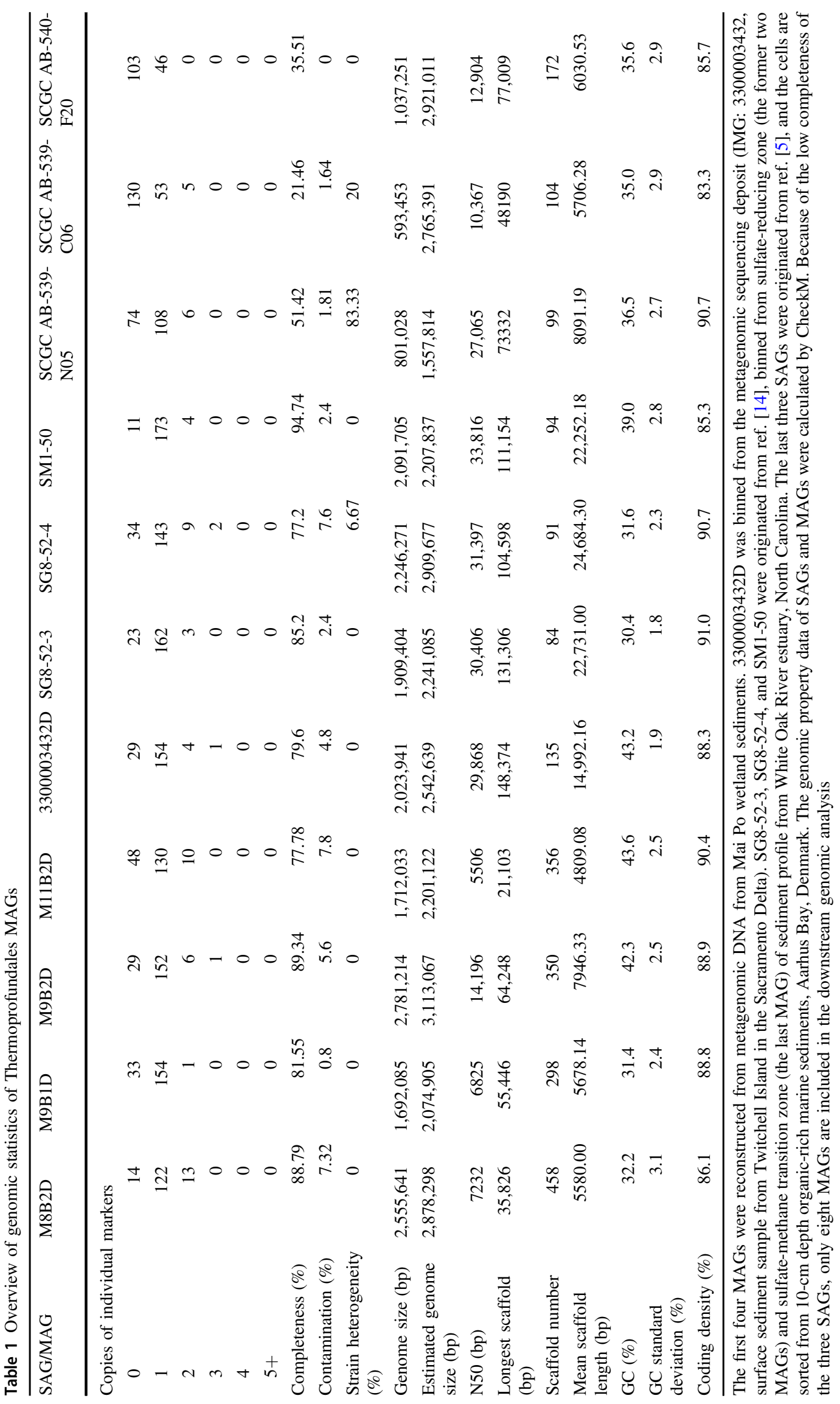




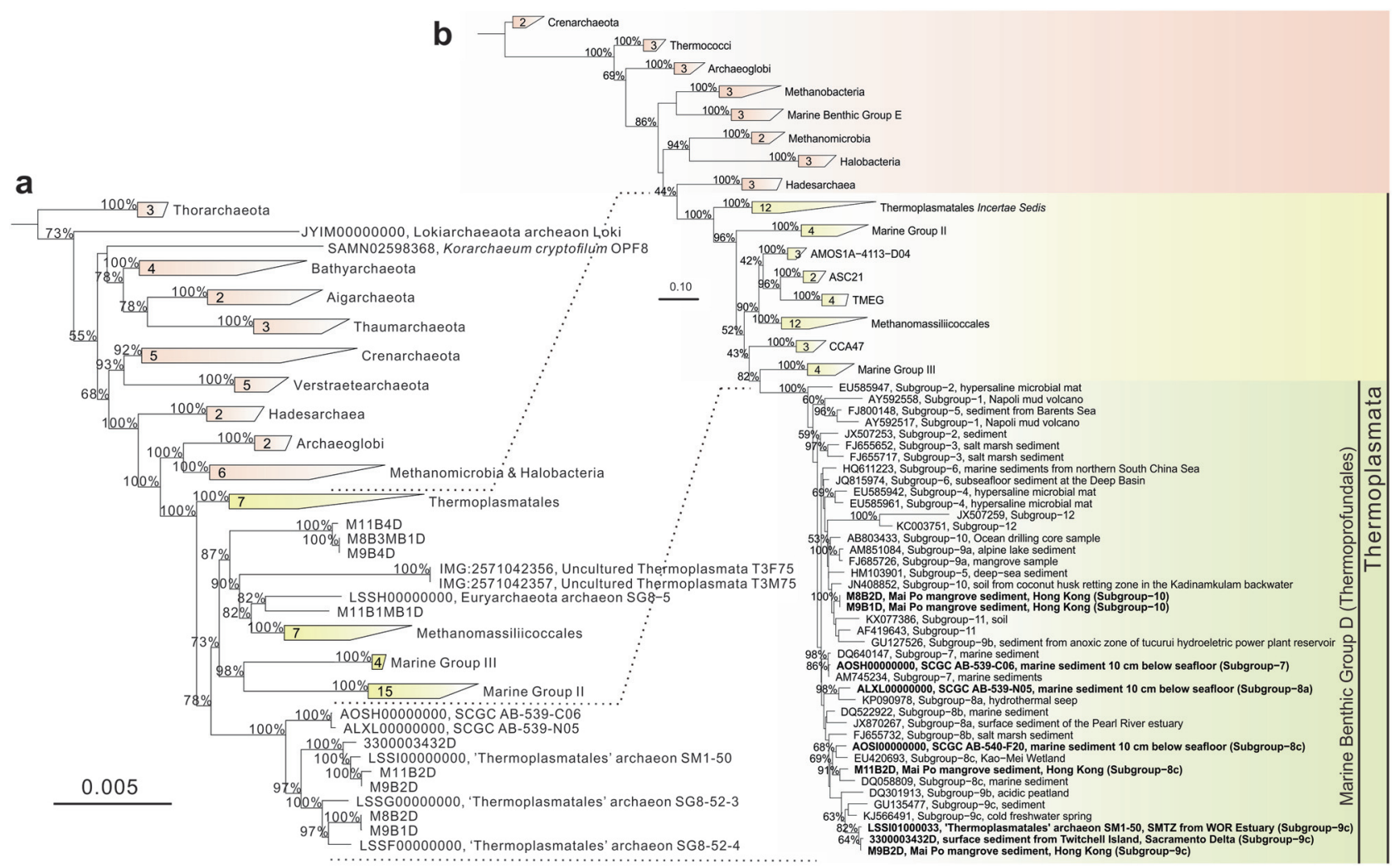

Fig. 3 Phylogenetic tree placing Thermoprofundales archaea into the Thermoplasmata. a Phylogenetic tree based on 43 concatenated markers. This concatenated protein alignment for phylogenomic reconstruction was obtained from the intermediate files in the process of placing MAGs and reference genomes into the reference tree by CheckM. b Phylogenetic tree based on 16S rRNA gene sequences of representative Thermoprofundales archaeon sequences from each

distribution, and another group as narrow lineages of rare/ less abundant distribution (Fig. 2a). This phenomenon is consistent with the macroecological concept "jack-of-alltrades is master of all", stating that cosmopolitan lineages could tolerate a wide range of environments and utilize a wide range of resources or commonly shared resources to become locally abundant in all environments [47-50]. The MBG-D archaea group is the second most frequent archaeal lineage, with 124/177 occurrences and $13.3 \%$ of relative abundance of 16S rRNA gene sequences in these studies, which outnumbers Thermoplasmatales $(98 / 177,6.5 \%)$ and other Thermoplasmata $(5 / 177,3.1 \%)$, suggesting that MBG-D archaea are a ubiquitous sedimentary archaeal lineage with an important ecological significance. The dispersion indices of archaeal lineages were plotted to test whether they follow a stochastic distribution (Poisson model) by comparing to a $0.5 \%$ confidence limit of chisquare distribution (Fig. 2b). The nine satellite lineages fell below the confidence limit, while the rest (the core lineages) were above it $(p<0.01)$, indicating a non-stochastic distribution among sedimentary environments. The MBG-D subgroup and the reference archaeal groups. SAG- or MAG-derived sequences are highlighted, and the dashed lines stand for the corresponding relationship between two trees. The method for tree reconstruction is detailed in Supplementary Information Note 2. The old names of "Thermoplasmatales" archaeon in both trees are used, but they are in fact affiliated to Thermoprofundales as figured out in this study

archaea, like the Bathyarchaeota investigated previously, appear to be core generalists non-randomly distributed across global sedimentary environments [47].

The MBG-D archaea from "MBG-D_over10_OTU table" were sorted into principal coordinates analysis (PCoA) ordination to look for associations between specific subgroups and particular environmental conditions (Fig. 2c). The salinity and environment category have large explanatory effects on the PCoA ordination with significant supports, and seep condition also shows significant influence on the PCoA ordination. These results indicate that salinity, habitat (also related to salinity), and seep condition have large influences on MBG-D archaea distribution. As evident in the ILs (the subgroups significantly associated with specific environments with statistical support) (Fig. 1), this probably results from the adaptation of particular subgroups to their corresponding eco-niches. Nevertheless, the potential physiological backgrounds of different subgroups, which might cause the different adaptation patterns, remain elusive. More studies on genomic and physiological profiles of MBG-D subgroups are encouraged to address their 
Table 2 Proposed taxonomic level based on sequence identity range

\begin{tabular}{|c|c|c|c|c|}
\hline Taxonomic group & Alternative name & $\begin{array}{l}\text { Median sequence } \\
\text { identity }^{\mathrm{a}}\end{array}$ & $\begin{array}{l}\text { Median sequence } \\
\text { identity }^{\mathrm{b}}\end{array}$ & $\begin{array}{l}\text { Proposed taxonomic level based on } \\
\text { sequence identity range }\end{array}$ \\
\hline Bathyarchaeota & $\begin{array}{l}\text { Miscellaneous Crenarchaeotal Group } \\
\text { (MCG) }\end{array}$ & 82.6 & 78.2 & Phylum \\
\hline Lokiarchaeota & Marine Benthic Group B (MBG-B) & 87.1 & 81.2 & Phylum-Class \\
\hline MSBL1 & Persephonarchaea (proposed) & 89.2 & 86.9 & Class-Order \\
\hline WSA2 & Candidatus Methanofastidiosa & 75.6 & 71.5 & Phylum \\
\hline Hadesarchaea & $\begin{array}{l}\text { South African Gold Mine Euryarchaeotic } \\
\text { Group (SAGMEG) }\end{array}$ & 86.7 & 82.8 & Class \\
\hline Thermoprofundales & Marine Benthic Group D (MBG-D) & 91.8 & 88.1 & Order-Family \\
\hline Marine Group II & Thalassoarchaea (proposed) & 80.0 & 77.3 & Phylum \\
\hline Marine Group III & Pontarchaea (proposed) & 90.0 & 78.4 & Class-Order \\
\hline
\end{tabular}

${ }^{a}$ Median sequence identity of representative sequences with 0.97 similarity cutoff from SILVA database with sequence length $>1400 \mathrm{bp}$ and pintail value $>75$ (Aug 10, 2017 updated). The sequence identity matrices for individual taxonomic group were calculated by BioEdit with default settings [87]

${ }^{b}$ Median sequence identity of representative sequences with 0.97 similarity cutoff from SILVA database with sequence length $>1200$ bp and pintail value $>75$ (Aug 10, 2017 updated)

${ }^{\mathrm{c}}$ The sequence identity ranges for different taxonomic levels are according to ref. [55]

adaptive strategies toward different environmental conditions.

\section{Co-occurrence network of sedimentary MBG-D archaea}

The co-occurrence network depicts potential closeinteracting or niche-sharing relationships in which MBG$\mathrm{D}$ archaea could be involved, by showing co-occurring patterns between OTUs with strong and significant correlations (Fig. 2d-f). The $C$-score test has indicated that the observed network (205 nodes and 571 edges) rejects the null model hypothesis of random co-occurrence, indicating that the observed network has fewer co-occurrences than expected by chance (containing segregated nodes in selective modules) (Supplementary Information Note 3). By comparing to 1000 times simulated ER random networks, the observed network has "small-world" properties, which means that, in this observed "small-world" network, nodes are more connected than in an identically sized random network [51]. Meanwhile, an MD (modularity degree) value $>0.4$ suggests that the observed network has a modular structure [52]. The modules are suggested as segregated functional/ecological niches [53].

The MBG-D archaea have the fourth most abundant nodes $(15.03 \%)$ in the observed network, occurring in 4 out of the 6 major modules. The exceptions are modules M3 and M5, which mainly represent assemblages of Thaumarchaeota and Bathyarchaeota (Fig. 2d, e). The cooccurrence incidences suggest that Lokiarchaeota have the highest non-random $\left(O / R_{\mathrm{ER}}\right.$ and $\left.O / R_{\mathrm{Ther}}\right)$ association with MBG-D archaea. Meanwhile, Hadesarchaea also show significant non-random association with MBG-D archaea in the network (Fig. 2e). This relationship could probably result from a niche overlap, rather than a synergistic/syntrophic relationship. However, as with Lokiarchaeota, these associations are present in all modules containing these two lineages (Fig. 2d, e), and two MBG-D archaea subgroups from non-saline and saline origins (Fig. 2f) are also associated with Lokiarchaeota, which might support a potential synergistic/syntrophic relationship between MBG-D and Lokiarchaeota.

\section{Genomic properties, definition, and description of Thermoprofundales (MBG-D)}

The obtained metagenomic sequencing data include three libraries of sizes 91.0, 88.6, and 86.0 gigabases for MaiPo8, MaiPo-9, and MaiPo-11, respectively. Four MBG-D archaeal MAGs (M11B2D, 5.0× coverage; M8B2D, 12.0× coverage; M9B1D, 48.0x coverage; and M9B2D, 23.5× coverage) with genome completeness $>75 \%$ were retrieved by metagenomic binning (Table 1). All the MBG-D MAGs resolved from Mai Po wetland and IMG deposited metagenomes had high genome completeness and low contamination and strain heterogeneity, compared to former MAGs and SAGs [5, 14].

Phylogenetic analyses of both the 43 concatenated markers and 16S rRNA genes confirmed the placement of MBG-D archaea into Class Thermoplasmata, within Phylum Euryarchaeota (Fig. 3). Furthermore, the sequence similarities among all available 16S rRNA genes of MBG-D archaea indicated that this archaeal group should be proposed as an order rather than a class (Izemarchaea) 

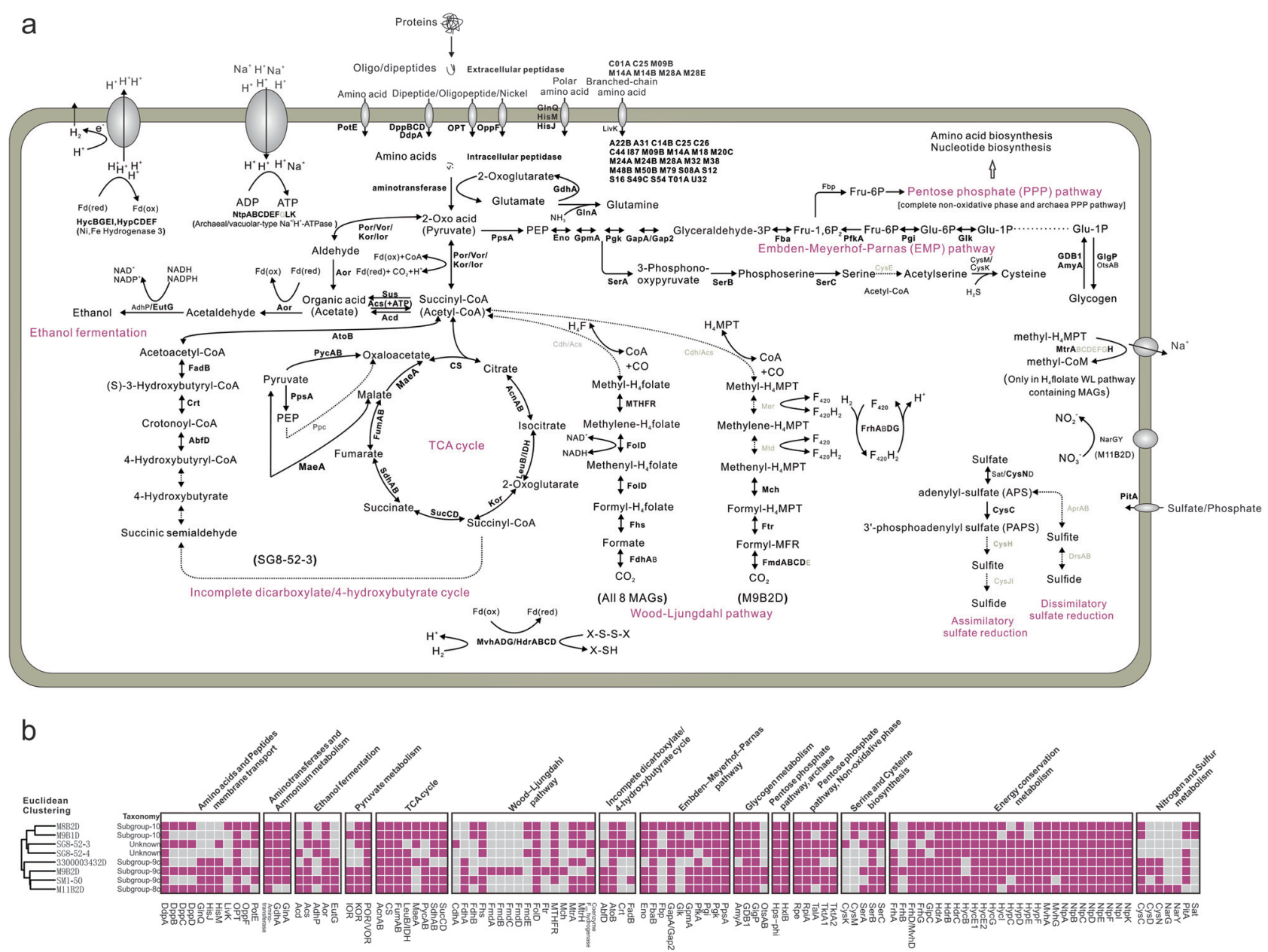

Fig. 4 Reconstructed key metabolic pathways of Thermoprofundales based on eight MAGs (a). Enzymes identified in over half of the eight MAGs are labeled in bold, those identified in less than half of the eight MAGs are labeled normally, and those for which no genes were found in any MAGs are labeled in gray. The extracellular and intracellular peptidases listed are those occurring in more than six out of the eight MAGs. Pathways for which no genes were found are drawn with dashed lines. The $\mathrm{H}_{4}$ MPT $\mathrm{C}_{1}$ carrier-dependent WL pathway and incomplete dicarboxylate/4-hydroxybutyrate cycle are depicted according to MAG M9B2D and SG8-52-3, respectively. Euclideanclustering-based gene presence heatmap (b). The presence/absence of protein encoding genes are represented by the heatmap, and the Euclidean clustering among all the MAGs was based on the presenceabsence binary matrix of the metabolic genes according to sequence identity range (Table 2) [19, 54, 55]. Based on the above genome properties and phylogeny of MBG-D archaea, we hereby proposed this uncultured archaeal group as a new archaeal order, Thermoprofundales (Ther.mo.pro.fund.a'les. N.L. suff. -ales ending designing an order name; N.L. masc. pl. n. Thermoprofundales, the order denoting MBG-D archaea, affiliated to Thermoplasmata). This nomenclature of Thermoprofundales is derived from the class name of Thermoplasmata, which is represented by the thermophilic acidophilic strains of Thermoplasma and Picrophilus [56]. Since Thermoprofundales was first discovered in marine benthic sediments with various widespread distribution and enigmatic ecological roles, L. adj. profundus could generally describe its living preference [9].

\section{Wood-Ljungdahl (WL) pathway with two types of $C_{1}$ carrier}

Thermoprofundales MAGs in this study mainly come from subgroup 8c, 9c, and 10 (Figs. 3 and 4), which cover both saline and non-saline ILs. As genome representatives, the MAGs enable us to provide comprehensive insights on the metabolic properties of Thermoprofundales. The presence/ absence matrix of metabolic genes showed two general clusters (Fig. 4); however, the LEfSe (linear discriminant analysis effect size) analysis indicated that no significant gene repertoire distribution associated with different subgroups was detected (Supplementary Information Note 5). Therefore, we integrated all the MAGs in the following genomic content analysis. 
Among the eight available MAGs, M9B2D was the only one containing genes for the tetrahydromethanopterin $\left(\mathrm{H}_{4} \mathrm{MPT}\right)-\mathrm{WL}$ pathway. This MAG contained putative genes for subunits of the formyl-methanofuran dehydrogenase (Fmd) in the carbonyl-branch of the WL pathway and the formyl transferase (Frt) and 5,10-methenyl- $\mathrm{H}_{4} \mathrm{MPT}$ cyclohydrolase (Mch) of the methyl-branch but lacked the rest protein-coding genes (Fig. 4). On the other hand, all the eight Thermoprofundales MAGs (including M9B2D) contained putative genes for intermediate enzymes in methylbranch of $\mathrm{H}_{4}$ folate-WL pathway, similar to typical bacterial acetogens [57]. No genes for CO-dehydrogenase/acetylCoA synthase (Cdh/Acs) were identified in all MAGs, except for a putative CdhA gene (converting $\mathrm{CO}_{2}$ to $\mathrm{CO}$ ) in SG8-52-3. Cdh/Acs is important for the carbonyl branch of WL pathway for reducing $\mathrm{CO}_{2}$ to $\mathrm{CO}$ and combining $\mathrm{CO}$ with a methyl residue to produce acetyl-CoA [58, 59]. Notably, genes encoding $\mathrm{Cdh} /$ Acs complex within the WL pathway are commonly absent in many available genomes affiliated to Thermoplasmatales, MG-II, and MG-III (Supplementary Information Note 5), probably because their $\mathrm{Cdh} / \mathrm{Acs}$ complexes share less sequence similarity with those from other lineages of Euryarchaeota and TACK superphylum.

\section{Incomplete dicarboxylate/4-hydroxybutyrate cycle}

Several Thermoprofundales MAGs encoded enzymes involved in the hydroxybutyrate (HB) part of the dicarboxylate/4-hydroxybutyrate cycle (abbreviated as DC/4-HB or dicarboxylate/hydroxybutyrate cycle), which converts one succinyl-CoA molecule through 4-HB to two acetylCoA molecules. Among the MAGs, SG8-52-3 had the most complete inferred pathway for the HB part (Fig. 4). However, it still lacked succinyl-CoA reductase, succinic semialdehyde reductase (NADPH), and 4-hydroxybutyrate-CoA ligase, which would catalyze the steps from succinyl-CoA to 4-hydroxybutyryl-CoA. SG8-52-3 does contain a candidate 4-hydroxybutyryl-CoA dehydratase gene, the maker gene for catalyzing the radical-mediated dehydration from 4-hydroxybutyrate-CoA [60, 61]. Similar genomic evidence could be found in Archaeoglobus fulgidus DSM 4304, A. fulgidus DSM 8774 [60, 62] and Ignicoccus hospitalis KIN4/I [63], where their genomes contain nearly all genes in the DC/4-HB cycle but all lack potential genes of succinyl-CoA reductase and succinic semialdehyde reductase (NADPH), similar to Thermoprofundales. Thus evidence suggests that Thermoprofundales might have the effective DC/4-HB cycle and the apparently missing genes may be too divergent from the known ones to be detected by the methods used. Nevertheless, enzymatic analyses and substrate incorporation experiments are required to further confirm the function of the DC/4-HB cycle.
Interestingly, SG8-52-3 is predicted to encode an alternative decarboxylation pathway $[60,63]$. It apparently lacks a phosphoenolpyruvate carboxylase, which produces oxaloacetate and fixes one molecule of $\mathrm{CO}_{2}$. Alternatively, it could use one of the following bypasses: (i) converting pyruvate to malate via the catalysis of (S)-Malate:NAD(P) ${ }^{+}$ oxidoreductase, and then using the subsequent tricarboxylic acid (TCA) cycle to generate succinyl-CoA; (ii) directly carboxylating pyruvate to oxaloacetate via the catalysis of pyruvate carboxylase, and then applying the normal TCA cycle to generate succinyl-CoA. Genes potentially encoding both the DC/4-HB cycle and the TCA cycle were found in nearly all the Thermoprofundales MAGs and were especially complete in SG8-52-3, which is similar to the scenario in the Thermoproteales genome [64]. They could both be used for autotrophic $\mathrm{CO}_{2}$ fixation; however, when both exist in one genome, the $\mathrm{DC} / 4-\mathrm{HB}$ cycle is expected to operate actively rather than the reductive TCA cycle in Thermoproteales and Desulfurococcales [64]. In our case, the TCA cycle may operate only in the oxidative direction for heterotrophic acetyl-CoA utilization, because that the reductive TCA cycle markers $a c l A B$ and $\operatorname{frd} A B$ were not found in any of the MAGs [65]. Combined with the above analysis, the WL pathway and DC/4-HB cycle within Thermoprofundales probably both participate in the autotrophic direction.

\section{Extracellular and intracellular peptidases, amino acid, and carbohydrate metabolism}

Consistent with earlier studies, putative genes for clostripain (C11), gingipain (C25), interpain (C10), and legumain (C13) were each found in at least one of these MAGs [5, 14]. Possible genes for other extracellular peptidases, including collagenase H (M09B), carboxypeptidase A1\&E (M14A \& B), and aminopeptidase S\&Ap1 (M28A \& E) were each found in at least six of eight MAGs (Supplementary Table S3). Amino acid/polar amino acid and oligopeptide transporters and ABC-type dipeptide/ oligopeptide/nickel transport systems were discovered in at least half of eight MAGs (Supplementary Table S4). A candidate LivK, the substrate binding protein of the branched-chain amino acid transport systems, could only be identified in M8B2D and M9B2D (Supplementary Table S4). There were 26 types of intracellular peptidases discovered in the at least 6 of the 8 MAGs (Supplementary Table S3). A variety of aminotransferases were present for transferring amino-groups from amino acids to 2oxoglutarate and generating glutamate (Supplementary Table S4). Pyruvate/ketoisovalerate:ferredoxin oxidoreductase (Por/Vor) and 2-oxoglutarate/2-oxoacid:ferredoxin oxidoreductase (Kor) were present in six to eight MAGs, and indolepyruvate:ferredoxin oxidoreductase (Ior) was 


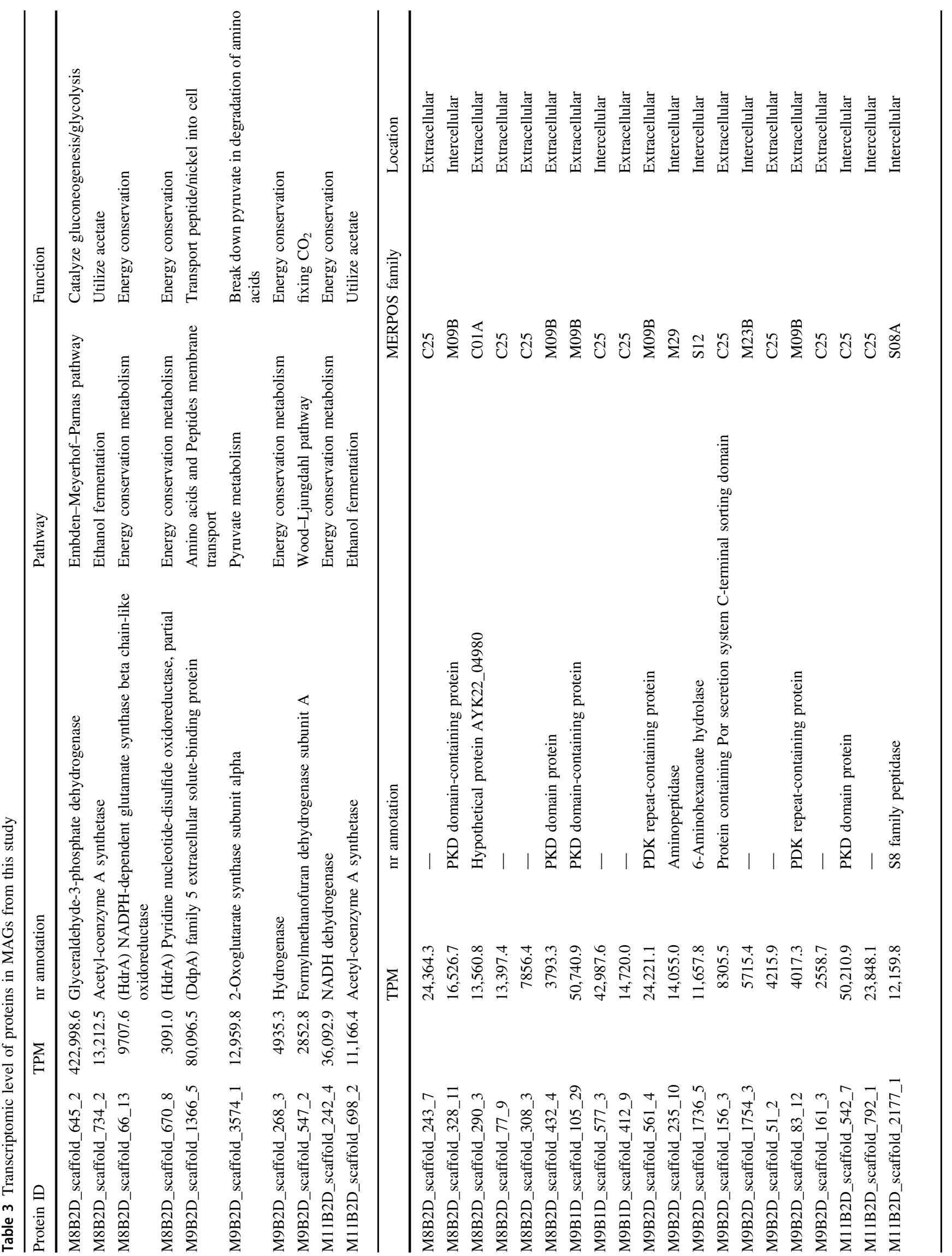


present in four out of the eight MAGs. All of them may be responsible for breaking down amino acids [5, 66] and mediate the complete pathways of transporting and breaking down proteins to acetyl-CoA, generating intermediate $\mathrm{CO}_{2}$, and transferring electrons to oxidized ferredoxins for energy recycling (Fig. 4).

Interestingly, none of the Thermoprofundales MAGs contained identifiable genes for ABC-type sugar transport system proteins or featured proteins of carbohydrate assimilation, highlighting that using proteins rather than carbohydrates for carbon and energy sources may be a common metabolic property of Thermoprofundales [14]. On the one hand, acetate from extracellular import and intermediates in the protein degradation pathway could be converted into acetyl-CoA by AMP-forming acetyl-CoA synthetase (EC: 6.2.1.1). On the other hand, acetate could be produced by ATP-producing acetyl-CoA synthetase (EC: 6.2.1.13) and acetaldehyde could be produced from acetate at the cost of reduced ferredoxins by aldehyde:ferredoxin oxidoreductase (Aor) (Fig. 4). Six out of the eight MAGs contained putative genes for iron-containing alcohol dehydrogenase family proteins EutG or AdhP, and SM1-50 has both of them (Fig. 4). The EutG and AdhP candidates are both of very low $(<50 \%)$ sequence identity with their homologs in the nr database and form a branch phylogenetically distinct from other bacterial clades in the phylogenetic tree (Supplementary Information Note 5). Both the conserved domain and functional site analyses suggest that they acquire the ethanol-producing functions (Supplementary Information Note 5). Over half of the eight Thermoprofundales MAGs contained a complete EmbdenMeyerhof-Parnas (EMP) pathway for both gluconeogenesis and glycolysis. They encoded nearly all the key genes for the non-oxidative phase of pentose phosphate pathway, in which ribose-5P, the important intermediate for both DNA and RNA biosynthesis, and erythrose-4-P, the precursor of aromatic amino acids, were produced. The Thermoprofundales MAGs also encoded serine and cysteine biosynthesis pathways, branching off from the gluconeogenesis direction of the EMP pathway. This suggests that Thermoprofundales maintain a well-established nucleic acid and amino acid anabolic metabolism for essential cell life activities [67].

\section{Nitrogen and sulfur metabolisms}

The majority of the eight MAGs contained phosphate/sulfate permeases for transporting phosphate/sulfate into cells (Fig. 4). Putative genes for the first two steps of assimilatory sulfate reduction, reducing sulfate to sulfite via adenylylsulfate and $3^{\prime}$-phosphoadenylyl sulfate, could be found in the majority of the eight MAGs. However, the two steps of reducing sulfite to sulfide via the catalysis of $\mathrm{CysH}$ and 
CysJI are missing. This indicates that Thermoprofundales could probably depend on sulfate assimilation rather than only depending on incorporating sulfur sources from sulfurcontaining peptides $[14,68]$, a strategy to assimilate more sulfur for biosynthesis from sulfate flux derived from upper oxic layers. M11B2D, resolved from intertidal mudflat sediment, contained putative nitrate reductase (NarGY) genes, indicating that Thermoprofundales from this econiche could participate in the initial step of denitrification or dissimilatory nitrate reduction to ammonia. The closest NarGY protein hits in the nr database were of Sulfuricurvum (bacterial) origin, instead of archaeal origin. Nevertheless, the nar gene was inferred to emerge before the divergence of bacteria and archaea during the pre-oxic times, and horizontal gene transfer between archaea and bacteria could also blur the phylogenetic relationship between nar and 16S rRNA genes [69].

\section{Transcriptomic pattern}

After processing read quality control (read dereplication and low-quality read trimming as described above) and deleting rRNA reads, three metatranscriptomic libraries were obtained from three sediment samples, which are of sizes 8.4, 8.7, and 12.0 gigabases for MaiPo-8, MaiPo-9, and MaiPo-11, respectively. The transcripts of MAGs from this study reflect the expression level of certain pathways and genes (Table 3, Supplementary Table S5). Key genes of acetate and amino acid utilization and peptide transportation were effectively expressed, which corroborates the metabolic properties deduced from metagenomic analysis. Genes assigned to proteins involved in the WL pathway, EMP pathway, and energy conservation were also highly expressed, which indicates that pathways for cell function and genes for building cell structures were also active in Thermoprofundales. The extracellular and intracellular peptidases, including C01A, C14B, C25, M09B, and etc., were expressed in these MAGs. Specifically, M09B (collagenase) as one of the most abundant extracellular peptidases in Thermoprofundales genomes were also mostly expressed in all four mangrove MAGs (TPM ranging from 3793.3 to 50,740.9). Collagen is the most abundant and ubiquitous material making up the extracellular matrices of animals, and nearly $30 \%$ of the total protein of animals are made of collagens [70]. Many collagenolytic-proteasesecreting bacteria have been isolated from terrestrial and marine sediments [70]. It is suggested that collagen degradation by extracellular collagenolytic proteases from various environmental bacteria is an important biological process for the release of fixed nitrogen (such as that within animal carcasses) into the global nitrogen cycle [70]. The high expression of collagenase of Thermoprofundales suggests their role of utilizing detrital proteins in global sediments.

\section{Biogeochemical roles and metabolic summaries}

Genomic studies have suggested that Thermoprofundales could effectively use proteins for biosynthesis and cell activity but lack pathways for transporting and assimilating carbohydrates [14]. Since mangrove leaves and wood debris are mainly made of lignocellulose, their colonization and degradation by heterotrophic bacteria and fungi should release plant-derived oligosaccharides to mangrove sediments [71-73]. Although oligosaccharides might not be directly utilized by Thermoprofundales, they could be utilized by other heterotrophs to generate intermediates or products that would be subsequently utilized by Thermoprofundales. According to the above genomic studies and previous results [18], Thermoprofundales in the Mai Po wetland, which occupy $32 \%$ relative abundance among archaeal communities, could fuel the turnover of organic matter, especially for detrital proteins, together with other microbial heterotrophs to recycle and conserve nutrients and maintain microbe-nutrient-plant relationship and extensive food web of the ecosystem [71].

Given their predicted potential for protein remineralization and for $\mathrm{CO}_{2}$ fixation by the WL pathway or the dicarboxylate/4-hydroxybutyrate cycle, it is reasonable to suggest that the global distribution of Thermoprofundales may be due to their mixotrophic lifestyle (Figs. 2 and 4) $[5,14]$. Beyond that, they also appear to have acquired the ability to assimilate sulfur from sulfate or protein-derived sulfur compounds. This metabolic capacity may have enabled Thermoprofundales to effectively adapt to benthic sediment environments with various carbon substrate conditions, especially in the energy-limited deep subsurface $[5,14]$. Thermoprofundales could produce acetate and ethanol, subsequently providing small molecular substrates for heterotrophic microorganisms and acetoclastic methanogens. Acetogenesis is the energetically more favorable metabolic pathway for organic substrate utilization, so that Thermoprofundales may serve as effective organic matter transformers in benthic sediment environments $[74,75]$.

Genomic reconstruction has suggested that Lokiarchaeota might be not strictly autotrophic but could derive energy from acetogenesis on hydrogen, formate, or other organics $[76,77]$. Our results raise the possibility that Thermoprofundales and Lokiarchaeota might use similar substrates in both marine and terrestrial sedimentary environments, synergistically work for carbon remineralization from sediments, and produce more labile compounds for other microorganisms. Nevertheless, 
co-occurrence networks do not always effectively predict actual classical ecological networks, in which the interactions are represented by direct observations or experiment manipulations [78], thus the omics-based profiling and culture-dependent approaches are needed to further test and understand the potential synergistic/syntrophic relationship. Furthermore, Woesearchaeota AR20, Diapherotrites AR10 (DPANN superphylum), Thorarchaeota, and Lokiarchaeota all contain alcohol dehydrogenase for ethanol synthesis from acetyl-CoA and could produce acetate and ethanol as fermentation products $[79,80]$. Thermoprofundales, together with other major archaeal groups, such as Bathyarchaeota $[5,15,74,81]$, Thorarchaeota [82, 83], Lokiarchaeota [79, 84], Woesearchaeota [80, 82], Diapherotrites [80, 85], Marine Group II/III [86], and etc., have a wide spectrum of organic-matter-utilizing capacity and synergistically fuel the carbon turnover in natural environments, providing new microbial biogeochemical insights on the carbon and nutrient flow in global scale.

Acknowledgements We thank Ms. Yueping Pan for the laboratory assistance work and Ms. Kelly Lau for the sampling support. We also thank Professor Bernhard Schink (University of Konstanz) for the suggestion on the nomenclature of Thermoprofundales and Dr. Anyi Hu (Institute of Urban Environment, CAS) for the help on the R script of IoD plot. This study was funded by National Natural Science Foundation of China (No. 31622002, 91851105, 31600093, 31700430), Science and Technology Innovation Committee of Shenzhen (No. JCYJ20170818091727570), the Key Project of Department of Education of Guangdong Province (No.2017KZDXM071), and the RGC GRF of Hong Kong (No. 701913).

Author contributions ZZ, ML, and J-DG conceived this study, ZZ, YL, JP, and ML performed the metagenomic binning and genomic analysis. ZZ and ML wrote the manuscript, and YL, KGL, and JP contributed suggestions to the genomic analysis. ZZ collected samples and performed the physicochemical analyses. ZZ, YL, and JP contributed to the analyses in Supplementary Information, and $\mathrm{ZZ}$ wrote it. All authors were involved in the manuscript writing and approved the final edition of the manuscript.

Data availability The GenBank WGS master accession numbers for the four MBG-D MAGs from Mai Po wetland sediments of this study are MUGB00000000 (M8B2D), MUGC00000000 (M9B1D), MUGD00000000 (M9B2D), and MUGE00000000 (M11B2D), respectively. The GenBank WGS master accession number of the Thermoprofundales MAG 3300003432D, which was binned from metagenome IMG OID: 3300003432, is DQIQ00000000. The IMG OIDs for the three metagenomes are 3300009506 (MaiPo-8), 3300010413 (MaiPo-9), and 3300009509 (MaiPo-11), respectively. The transcriptomic reads are deposited in NCBI-SRA with the following accession numbers: SRR7284896 (MaiPo-8), SRR7284884 (MaiPo-9), and SRR7286715 (MaiPo-11).

\section{Compliance with ethical standards}

Conflict of interest The authors declare that they have no conflict of interest.
Open Access This article is licensed under a Creative Commons Attribution 4.0 International License, which permits use, sharing, adaptation, distribution and reproduction in any medium or format, as long as you give appropriate credit to the original author(s) and the source, provide a link to the Creative Commons license, and indicate if changes were made. The images or other third party material in this article are included in the article's Creative Commons license, unless indicated otherwise in a credit line to the material. If material is not included in the article's Creative Commons license and your intended use is not permitted by statutory regulation or exceeds the permitted use, you will need to obtain permission directly from the copyright holder. To view a copy of this license, visit http://creativecommons. org/licenses/by/4.0/.

\section{References}

1. Kallmeyer J, Pockalny R, Adhikari RR, Smith DC, D'Hondt S. Global distribution of microbial abundance and biomass in subseafloor sediment. Proc Natl Acad Sci USA. 2012;109:16213-6.

2. Lipp JS, Morono Y, Inagaki F, Hinrichs K-U. Significant contribution of Archaea to extant biomass in marine subsurface sediments. Nature. 2008;454:991-4.

3. Biddle JF, Lipp JS, Lever MA, Lloyd KG, Sorensen KB, Anderson R, et al. Heterotrophic Archaea dominate sedimentary subsurface ecosystems off Peru. Proc Natl Acad Sci USA. 2006;103:3846-51.

4. Fry JC, Parkes RJ, Cragg BA, Weightman AJ, Webster G. Prokaryotic biodiversity and activity in the deep subseafloor biosphere. FEMS Microbiol Ecol. 2008;66:181-96.

5. Lloyd KG, Schreiber L, Petersen DG, Kjeldsen KU, Lever MA, Steen AD, et al. Predominant archaea in marine sediments degrade detrital proteins. Nature. 2013;496:215-8.

6. Biddle JF, Fitz-Gibbon S, Schuster SC, Brenchley JE, House CH. Metagenomic signatures of the Peru Margin subseafloor biosphere show a genetically distinct environment. Proc Natl Acad Sci USA. 2008;105:10583-8.

7. Sorensen KB, Teske A. Stratified communities of active archaea in deep marine subsurface sediments. Appl Environ Microbiol. 2006;72:4596-603.

8. Teske A, Sørensen KB. Uncultured archaea in deep marine subsurface sediments: have we caught them all? ISME J. 2008;2:318.

9. Vetriani C, Jannasch HW, MacGregor BJ, Stahl DA, Reysenbach AL. Population structure and phylogenetic characterization of marine benthic archaea in deep-sea sediments. Appl Environ Microbiol. 1999;65:4375-84.

10. Inagaki F, Kuypers MMM, Tsunogai U, Ishibashi J-i, Nakamura $\mathrm{K}-\mathrm{i}$, Treude T, et al. Microbial community in a sediment-hosted $\mathrm{CO} 2$ lake of the southern Okinawa Trough hydrothermal system. Proc Natl Acad Sci USA. 2006;103:14164-9.

11. Holmkvist L, Ferdelman TG, Jørgensen BB. A cryptic sulfur cycle driven by iron in the methane zone of marine sediment (Aarhus Bay, Denmark). Geochim Cosmochim Acta. 2011;75:3581-99.

12. Borrel G, Lehours A-C, Crouzet O, Jézéquel D, Rockne K, Kulczak A, et al. Stratification of archaea in the deep sediments of a freshwater meromictic lake: vertical shift from methanogenic to uncultured archaeal lineages. PLoS ONE. 2012;7:e43346.

13. Swan BK, Ehrhardt CJ, Reifel KM, Moreno LI, Valentine DL. Archaeal and bacterial communities respond differently to environmental gradients in anoxic sediments of a California hypersaline lake, the Salton Sea. Appl Environ Microbiol. 2010;76:75768.

14. Lazar CS, Baker BJ, Seitz KW, Teske AP. Genomic reconstruction of multiple lineages of uncultured benthic archaea suggests 
distinct biogeochemical roles and ecological niches. ISME J. 2017;11:1118-29.

15. Lazar CS, Baker BJ, Seitz K, Hyde AS, Dick GJ, Hinrichs K-U, et al. Genomic evidence for distinct carbon substrate preferences and ecological niches of Bathyarchaeota in estuarine sediments. Environ Microbiol. 2016;18:1200-11.

16. Zhou Z, Chen J, Cao H, Han P, Gu J-D. Analysis of methaneproducing and metabolizing archaeal and bacterial communities in sediments of the northern South China Sea and coastal Mai Po Nature Reserve revealed by PCR amplification of $m c r A$ and $p m o A$ genes. Front Microbiol. 2015;5:789.

17. Liu Y, Zhou Z, Pan J, Baker BJ, Gu J-D, Li M. Comparative genomic inference suggests mixotrophic lifestyle for Thorarchaeota. ISME J. 2018;12:1021-31.

18. Zhou Z, Meng H, Liu Y, Gu J-D, Li M. Stratified bacterial and archaeal community in mangrove and intertidal wetland mudflats revealed by high throughput $16 \mathrm{~S}$ rRNA gene sequencing. Front Microbiol. 2017;8:2148.

19. Quast C, Pruesse E, Yilmaz P, Gerken J, Schweer T, Yarza P, et al. The SILVA ribosomal RNA gene database project: improved data processing and web-based tools. Nucleic Acids Res. 2013;41:D590-D596.

20. Pruesse E, Peplies J, Gloeckner FO. SINA: accurate highthroughput multiple sequence alignment of ribosomal RNA genes. Bioinformatics. 2012;28:1823-9.

21. Ludwig W, Strunk O, Westram R, Richter L, Meier H, Yadhukumar, et al. ARB: a software environment for sequence data. Nucleic Acids Res. 2004;32:1363-71.

22. Caporaso JG, Kuczynski J, Stombaugh J, Bittinger K, Bushman FD, Costello EK, et al. QIIME allows analysis of high-throughput community sequencing data. Nat Methods. 2010;7:335-6.

23. Miller MA, Pfeiffer W, Schwartz T. Creating the CIPRES science gateway for inference of large phylogenetic trees. Gateway computing environments workshop (GCE). New Orleans, Louisiana, USA: IEEE; 2010.

24. Trifinopoulos J, Nguyen L-T, von Haeseler A, Minh BQ. W-IQTREE: a fast online phylogenetic tool for maximum likelihood analysis. Nucleic Acids Res. 2016;44:W232-W235.

25. Letunic I, Bork P. Interactive tree of life (iTOL): an online tool for phylogenetic tree display and annotation. Bioinformatics. 2007;23:127-8.

26. Dufrene M, Legendre P. Species assemblages and indicator species: the need for a flexible asymmetrical approach. Ecol Monogr. 1997;67:345-66.

27. Barberán A, Bates ST, Casamayor EO, Fierer N. Using network analysis to explore co-occurrence patterns in soil microbial communities. ISME J. 2011;6:343.

28. Ju F, Xia Y, Guo F, Wang Z, Zhang T. Taxonomic relatedness shapes bacterial assembly in activated sludge of globally distributed wastewater treatment plants. Environ Microbiol. 2014;16:2421-32.

29. Csardi G, Nepusz T. The igraph software package for complex network research. Inter Complex Syst. 2006;1695:1-9.

30. Bastian M, Heymann S, Jacomy M. Gephi: an open source software for exploring and manipulating networks. ICWSM Conf. 2009;8:361-2.

31. Oksanen J, Kindt R, Legendre P, O'Hara B, Stevens MHH, Oksanen MJ, et al. The vegan package. Community ecology package. 2007;631-7.

32. Peng Y, Leung HC, Yiu SM, Chin FY. IDBA-UD: a de novo assembler for single-cell and metagenomic sequencing data with highly uneven depth. Bioinformatics. 2012;28:1420-8.

33. Markowitz VM, Chen I-MA, Chu K, Szeto E, Palaniappan K, Grechkin $\mathrm{Y}$, et al. IMG/M: the integrated metagenome data management and comparative analysis system. Nucleic Acids Res. 2012;40:D123-9.
34. Wu Y-W, Tang Y-H, Tringe SG, Simmons BA, Singer SW. MaxBin: an automated binning method to recover individual genomes from metagenomes using an expectation-maximization algorithm. Microbiome. 2014;2:1-18.

35. Parks DH, Imelfort M, Skennerton CT, Hugenholtz P, Tyson GW. CheckM: assessing the quality of microbial genomes recovered from isolates, single cells, and metagenomes. Genome Res. 2015;25:1043-55.

36. Bushnell, B. BBMap: A Fast, Accurate, Splice-Aware Aligner. Lawrence Berkeley National Laboratory. LBNL Report \#: LBNL7065E. Retrieved from https://escholarship.org/uc/item/1h3515gn 2014.

37. Huerta-Cepas J, Szklarczyk D, Forslund K, Cook H, Heller D, Walter MC, et al. eggNOG 4.5: a hierarchical orthology framework with improved functional annotations for eukaryotic, prokaryotic and viral sequences. Nucleic Acids Res. 2016;44:D286-93.

38. Hyatt D, Chen G-L, LoCascio PF, Land ML, Larimer FW, Hauser LJ. Prodigal: prokaryotic gene recognition and translation initiation site identification. BMC Bioinformatics. 2010;11:119.

39. Kanehisa M, Sato Y, Morishima K. BlastKOALA and GhostKOALA: KEGG tools for functional characterization of genome and metagenome sequences. J Mol Biol. 2016;428:726-31.

40. Pruitt KD, Tatusova T, Maglott DR. NCBI Reference Sequence (RefSeq): a curated non-redundant sequence database of genomes, transcripts and proteins. Nucleic Acids Res. 2005;33:D501-4.

41. Quevillon E, Silventoinen V, Pillai S, Harte N, Mulder N, Apweiler $\mathrm{R}$, et al. InterProScan: protein domains identifier. Nucleic Acids Res. 2005;33:W116-20.

42. Rawlings ND, Barrett AJ, Finn R. Twenty years of the MEROPS database of proteolytic enzymes, their substrates and inhibitors. Nucleic Acids Res. 2016;44:D343-50.

43. Bagos PG, Tsirigos KD, Plessas SK, Liakopoulos TD, Hamodrakas SJ. Prediction of signal peptides in archaea. Protein Eng Des Sel. 2009;22:27-35.

44. Nancy YY, Wagner JR, Laird MR, Melli G, Rey S, Lo R, et al. PSORTb 3.0: improved protein subcellular localization prediction with refined localization subcategories and predictive capabilities for all prokaryotes. Bioinformatics. 2010;26:1608-15.

45. Hug LA, Baker BJ, Anantharaman K, Brown CT, Probst AJ, Castelle CJ, et al. A new view of the tree of life. Nat Microbiol. 2016;1:16048.

46. Kopylova E, Noé L, Touzet H. SortMeRNA: fast and accurate filtering of ribosomal RNAs in metatranscriptomic data. Bioinformatics. 2012;28:3211-7.

47. Fillol M, Auguet J-C, Casamayor EO, Borrego CM. Insights in the ecology and evolutionary history of the Miscellaneous Crenarchaeotic Group lineage. ISME J. 2016;10:665-77.

48. Brown JH. On the relationship between abundance and distribution of species. Am Nat. 1984;124:255-79.

49. Gaston KJ, Blackburn TM, Lawton JH. Interspecific abundancerange size relationships: an appraisal of mechanisms. J Anim Ecol. 1997;66:579-601.

50. Gaston KJ, Blackburn TM, Greenwood JJ, Gregory RD, Quinn RM, Lawton JH. Abundance - occupancy relationships. J Appl Ecol. 2000;37:39-59.

51. Watts DJ, Strogatz SH. Collective dynamics of 'small-world'networks. Nature. 1998;393:440-2.

52. Newman ME. Modularity and community structure in networks. Proc Natl Acad Sci USA. 2006;103:8577-82.

53. Faust K, Raes J. Microbial interactions: from networks to models. Nat Rev Microbiol. 2012;10:538-50.

54. Adam PS, Borrel G, Brochier-Armanet C, Gribaldo S. The growing tree of Archaea: new perspectives on their diversity, evolution and ecology. ISME J. 2017;11:2407-25.

55. Yarza P, Yilmaz P, Pruesse E, Gloeckner FO, Ludwig W, Schleifer K-H, et al. Uniting the classification of cultured and 
uncultured bacteria and archaea using 16S rRNA gene sequences. Nat Rev Microbiol. 2014;12:635-45.

56. Boone DR, Castenholz RW, Garrity GM. Bergey's Manual of systematic bacteriology: Volume one: the Archaea and the deeply branching and phototrophic bacteria. New York: Springer-Verlag New York; 2001.

57. Sousa FL, Martin WF. Biochemical fossils of the ancient transition from geoenergetics to bioenergetics in prokaryotic one carbon compound metabolism. Biochim Biophys Acta. 2014;1837:964-81.

58. Fuchs G. Alternative pathways of carbon dioxide fixation: insights into the early evolution of life? Annu Rev Microbiol. 2011;65:631-58.

59. Maden BEH. Tetrahydrofolate and tetrahydromethanopterin compared: functionally distinct carriers in $\mathrm{C}_{1}$ metabolism. Biochem J. 2000;350:609-29.

60. Berg IA, Kockelkorn D, Ramos-Vera WH, Say RF, Zarzycki J, Hügler M, et al. Autotrophic carbon fixation in archaea. Nat Rev Microbiol. 2010;8:447-60.

61. Martins BM, Dobbek H, Cinkaya I, Buckel W, Messerschmidt A. Crystal structure of 4-hydroxybutyryl-CoA dehydratase: radical catalysis involving a [4Fe-4S] cluster and flavin. Proc Natl Acad Sci USA. 2004;101:15645-9.

62. Klenk H-P, Clayton RA, Tomb J-F, White O, Nelson KE, Ketchum KA, et al. The complete genome sequence of the hyperthermophilic, sulphate-reducing archaeon Archaeoglobus fulgidus. Nature. 1997;390:364-70.

63. Huber H, Gallenberger M, Jahn U, Eylert E, Berg IA, Kockelkorn D, et al. A dicarboxylate/4-hydroxybutyrate autotrophic carbon assimilation cycle in the hyperthermophilic Archaeum Ignicoccus hospitalis. Proc Natl Acad Sci USA. 2008;105:7851-6.

64. Ramos-Vera WH, Berg IA, Fuchs G. Autotrophic carbon dioxide assimilation in Thermoproteales revisited. J Bacteriol. 2009;191:4286-97.

65. Hügler M, Wirsen CO, Fuchs G, Taylor CD, Sievert SM. Evidence for autotrophic $\mathrm{CO}_{2}$ fixation via the reductive tricarboxylic acid cycle by members of the $\varepsilon$ subdivision of proteobacteria. $\mathbf{J}$ Bacteriol. 2005;187:3020-7.

66. Schut GJ, Menon AL, Adams MW. 2-keto acid oxidoreductases from Pyrococcus furiosus and Thennococcus litoralis. Methods Enzymol. 2001;331:144-58.

67. Madigan MT, Martinko JM, Bender KS, Buckley DH, Stahl DA. Brock biology of microorganisms. 14th ed. Boston: Pearson; 2015.

68. Erkel C, Kube M, Reinhardt R, Liesack W. Genome of Rice Cluster I archaea-the key methane producers in the rice rhizosphere. Science. 2006;313:370-2.

69. Cabello P, Roldán MD, Moreno-Vivián C. Nitrate reduction and the nitrogen cycle in archaea. Microbiology. 2004;150:3527-46.

70. Zhang Y-Z, Ran L-Y, Li C-Y, Chen X-L. Diversity, structures, and collagen-degrading mechanisms of bacterial collagenolytic proteases. Appl Environ Microbiol. 2015;81:6098-107.

71. Holguin G, Vazquez P, Bashan Y. The role of sediment microorganisms in the productivity, conservation, and rehabilitation of mangrove ecosystems: an overview. Biol Fertil Soils. 2001;33:265-78.
72. Moran MA, Hodson RE. Formation and bacterial utilization of dissolved organic carbon derived from detrital lignocellulose. Limnol Oceanogr. 1989;34:1034-47.

73. Steinke T, Barnabas A, Somaru R. Structural changes and associated microbial activity accompanying decomposition of mangrove leaves in Mgeni Estuary. S Afr J Bot. 1990;56:39-48.

74. He Y, Li M, Perumal V, Feng X, Fang J, Xie J, et al. Genomic and enzymatic evidence for acetogenesis among multiple lineages of the archaeal phylum Bathyarchaeota widespread in marine sediments. Nat Microbiol. 2016;1:16035.

75. Lever MA. Acetogenesis in the energy-starved deep biosphere - a paradox? Front Microbiol. 2011;2:284.

76. Sousa FL, Neukirchen S, Allen JF, Lane N, Martin WF. Lokiarchaeon is hydrogen dependent. Nat Microbiol. 2016;1:16034.

77. Spang A, Stairs C, Dombrowski N, Cáceres EF, Lombard J, Jørgensen SL, et al. Insights into the metabolic potential of Asgard archaea that have played a key role in the orgin of eukaryotes. International workshop on marine geomicrobiology - a matter of energy. Sandbjerg Manor, Denmark: Uppsala University; 2017.

78. Freilich MA, Wieters E, Broitman BR, Marquet PA, Navarrete SA. Species co-occurrence networks: Can they reveal trophic and non-trophic interactions in ecological communities? Ecology. 2018;99:690-9.

79. Cai M, Liu Y, Zhou Z, Yang Y, Pan J, Gu J-D, et al. Asgard archaea are diverse, ubiquitous, and transcriptionally active microbes. bioRxiv. 2018. https://doi.org/10.1101/374165.

80. Castelle CJ, Wrighton KC, Thomas BC, Hug LA, Brown CT, Wilkins MJ, et al. Genomic expansion of domain archaea highlights roles for organisms from new phyla in anaerobic carbon cycling. Curr Biol. 2015;25:690-701.

81. Zhou Z, Pan J, Wang F, Gu J-D, Li M. Bathyarchaeota: globally distributed metabolic generalists in anoxic environments. FEMS Microbiol Rev. 2018;42:639-55.

82. Liu X, Li M, Castelle CJ, Probst AJ, Zhou Z, Pan J, et al. Insights into the ecology, evolution, and metabolism of the widespread Woesearchaeotal lineages. Microbiome. 2018;6:102.

83. Seitz KW, Lazar CS, Hinrichs K-U, Teske AP, Baker BJ. Genomic reconstruction of a novel, deeply branched sediment archaeal phylum with pathways for acetogenesis and sulfur reduction. ISME J. 2016;10:1696-705.

84. Spang A, Caceres EF, Ettema TJG. Genomic exploration of the diversity, ecology, and evolution of the archaeal domain of life. Science. 2017;357:eaaf3883.

85. Youssef NH, Rinke C, Stepanauskas R, Farag I, Woyke T, Elshahed MS. Insights into the metabolism, lifestyle and putative evolutionary history of the novel archaeal phylum 'Diapherotrites'. ISME J. 2015;9:447-60.

86. Li M, Baker BJ, Anantharaman K, Jain S, Breier JA, Dick GJ. Genomic and transcriptomic evidence for scavenging of diverse organic compounds by widespread deep-sea archaea. Nat Commun. 2015;6:8933.

87. Hall TA. BioEdit: a user-friendly biological sequence alignment editor and analysis program for Windows 95/98/NT. Nucleic Acids Symp Ser. 1999;41:95-8. 